\title{
A Heart too Stiff to Beat: A Case of Familial Transthyretin Amyloidosis Cardiomyopathy
}

\author{
Yan Zhou ${ }^{1}$, Sameen Khalid ${ }^{1}$, Aamer Abbass ${ }^{1}$, Laura Hughes ${ }^{1}$, Marcos Hazday ${ }^{2}$ \\ 1. Internal Medicine Residency, Florida Hospital-Orlando 2. Cardiology, Florida Hospital-Orlando
}

$\square$ Corresponding author: Yan Zhou, dr.jessiezhou@gmail.com

Disclosures can be found in Additional Information at the end of the article

\section{Abstract}

Heart failure is a common clinical syndrome caused by a variety of cardiac diseases. We report a rare case of familial transthyretin amyloidosis cardiomyopathy to heighten the awareness of this rare but lethal cause of heart failure, as therapeutic interventions such as liver or heart transplant could be curative in selected patients.

Categories: Cardiology, Family/General Practice, Internal Medicine Keywords: transthyretin, amyloidosis, cardiomyopathy, liver transplant

\section{Introduction}

Amyloidosis refers to the extracellular deposition of insoluble fibrils that are composed of low molecular weight subunits of a variety of serum proteins. The three most common types of amyloidosis that can affect the heart are light-chain (AL) amyloidosis, familial or senile transthyretin amyloidosis (ATTR) and secondary (AA) amyloidosis [1]. Transthyretin (TTR) is a tetrameric serum protein that is synthesized primarily by the liver. Accumulation of TTR causes cell toxicity and tissue death. ATTR occurs in two types: non-mutated (senile/wild type) or mutated transthyretin with autosomal dominant inheritance (familial type) [2]. Familial ATTR is caused by any one of more than 100 mutations in the TTR gene, rendering it unstable and resulting in a tendency to misfold and produce amyloid which preferentially infiltrates the myocardium [3]. One of the most common mutations is caused by the Val122Ile mutation, which presents in three to four percent of the African-American and Afro-Caribbean populations. It is associated with a late-onset amyloid cardiomyopathy (CMP) characterized by progressive severe heart failure [4-6]. We report a case of familial ATTR cardiomyopathy to heighten the awareness of this rare but lethal cause of heart failure. Informed consent statement was obtained for this study.

Received 10/27/2016 Review began 12/28/2016 Review ended 03/07/2017 Published 03/20/2017

C) Copyright 2017

Zhou et al. This is an open access article distributed under the terms of the Creative Commons Attribution License CC-BY 3.0., which permits unrestricted use, distribution, and reproduction in any medium, provided the original author and source are credited.

\section{Case Presentation}

A 62-year-old Afro-Caribbean male was diagnosed with congestive heart failure with left ventricular ejection fraction (LVEF) 35\% two years ago at his home country and presented with progressing shortness of breath, paroxysmal nocturnal dyspnea, orthopnea. Other comorbidities included well-controlled type two diabetes mellitus and hypothyroidism. His home medications included furosemide, metformin, and levothyroxine. He has two family members who passed away from unknown heart disease after their 50s. Vital signs were significant for chronic borderline hypotension. Examination showed elevated jugular venous pressure, decreased breath sounds at the left base, and S3 heart sound. The electrocardiogram (EKG) showed low QRS voltage, but no ischemic changes [Figure 1]. 


\section{Cureus}

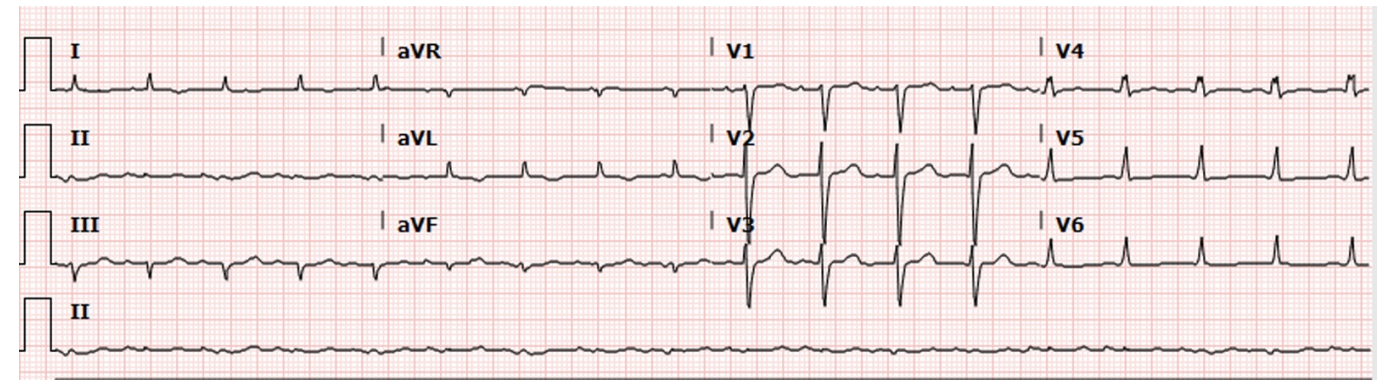

\section{FIGURE 1: Electrocardiogram}

Low QRS voltage without ischemic changes

Troponins were equivocal. The N-terminal pro-B-type natriuretic peptide (NT-proBNP) was elevated. Chest X-ray showed a moderate left pleural effusion with basilar atelectasis and mild pulmonary edema. Transthoracic echocardiography (TTE) revealed global hypokinesis with LVEF $16 \%$ and severe concentric biventricular hypertrophy [Figure 2].

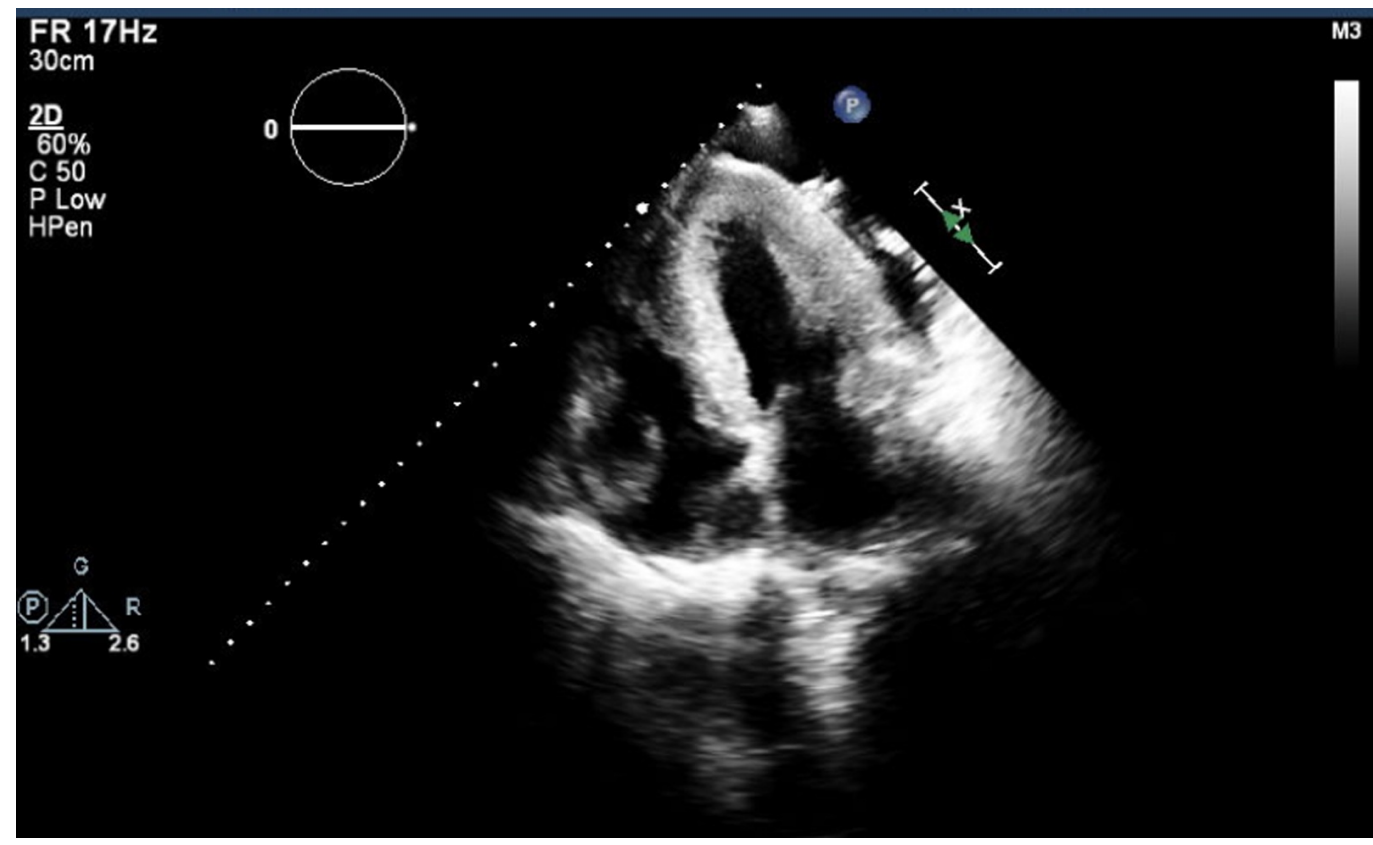

FIGURE 2: Transthoracic echocardiography four chamber view

Severe concentric biventricular hypertrophy

The patient was started on IV diuretics, dobutamine drip and had left-sided therapeutic thoracentesis with symptomatic improvement. Cardiac magnetic resonance imaging (MRI) with late gadolinium enhancement revealed diffusely enlarged concentrically thickened ventricles with increased myocardial signal suggestive of amyloid deposition by appearance [Figure 3]. 


\section{Cureus}

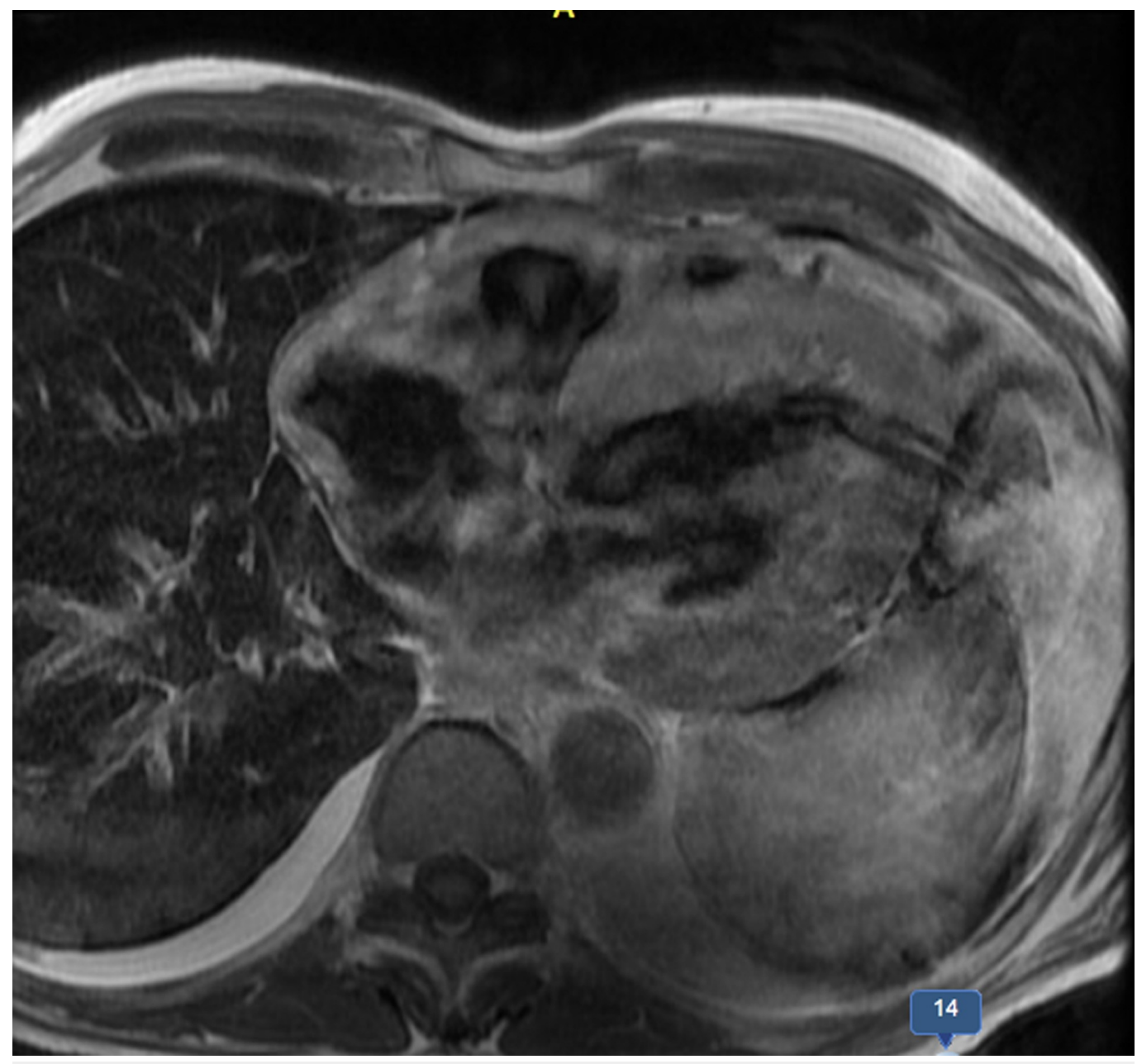

FIGURE 3: Cardiac MRI with late gadolinium enhancement

Diffusely enlarged concentrically thickened ventricles with increased myocardial signal suggestive of amyloid deposition by appearance

Left and right heart catheterization showed single-vessel coronary artery disases (CAD) isolated to the first obtuse marginal artery. Serum and urine immunofixations were negative. Right ventricular endomyocardial biopsy was obtained with amyloid subtype staining indicating ATTR [Figures 4-5]. 


\section{Cureus}
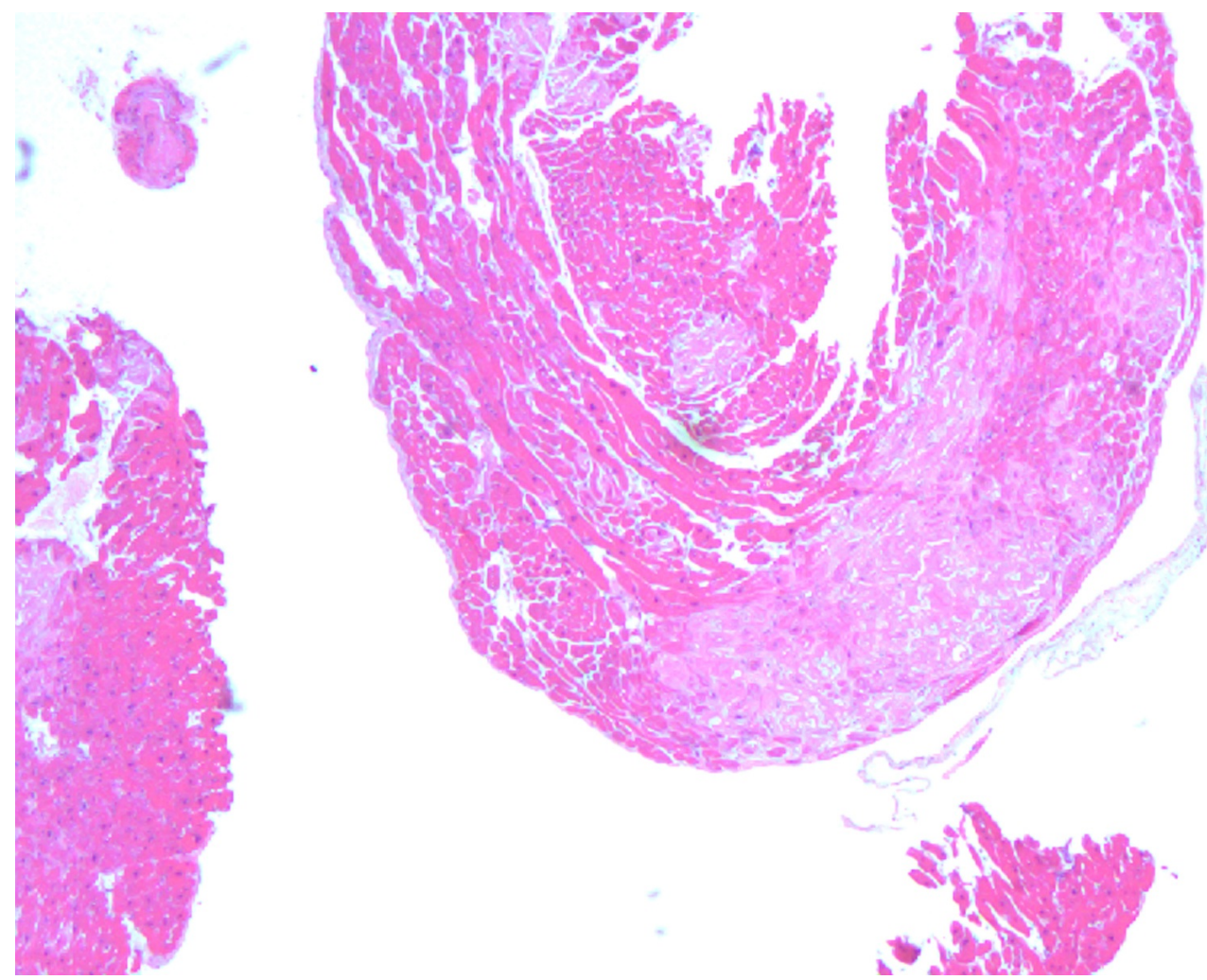

FIGURE 4: Haemotoxylin and eosin (H\&E) staining

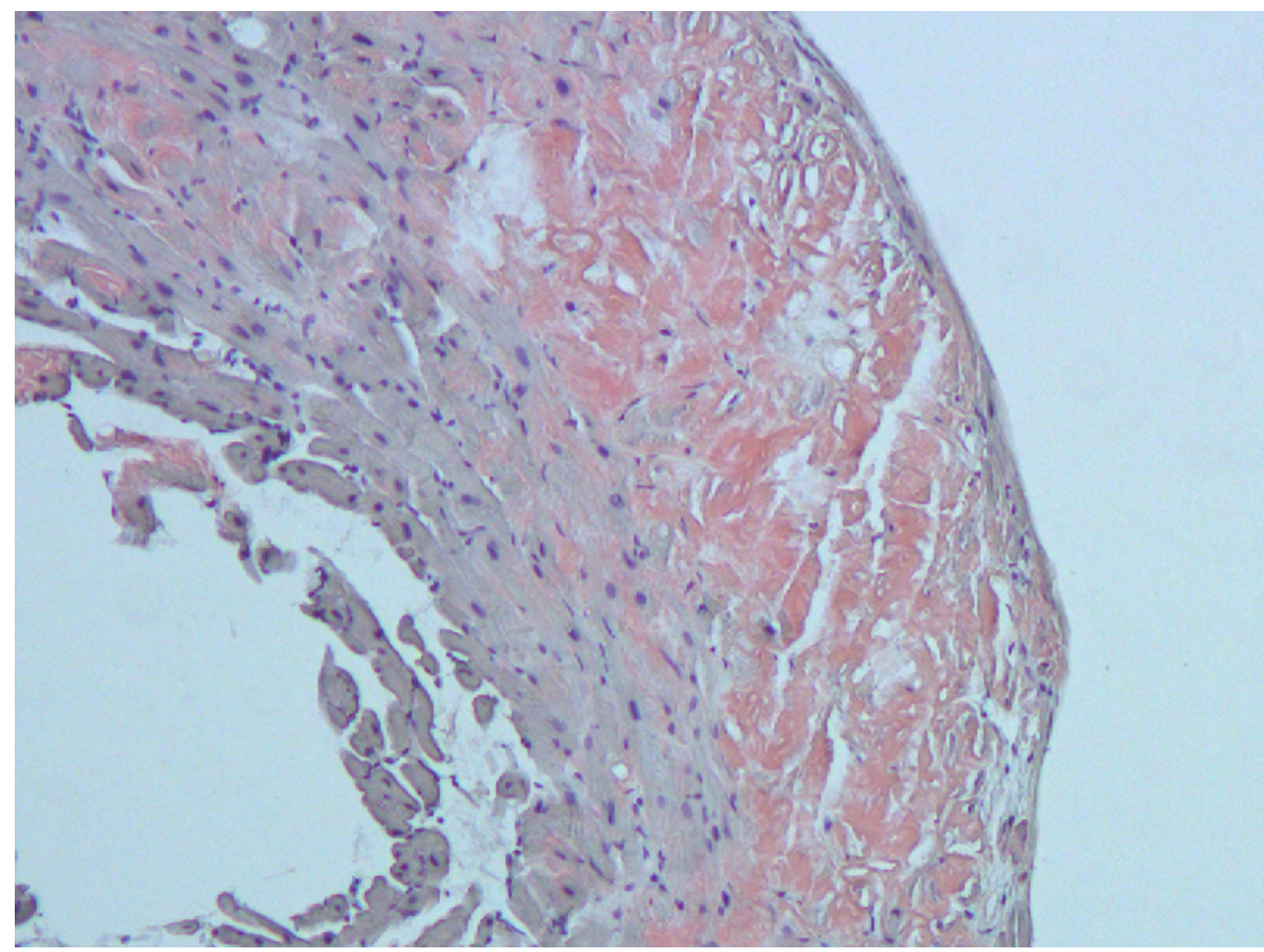

FIGURE 5: Congo red staining

The myocardium contains large patches of hyaline material that stains with Congo red, consistent with amyloid 
Further genetic sequencing analysis showed pathogenic Val122Ile mutation and confirmed familial ATTR cardiomyopathy. The patient developed several runs of asymptomatic nonsustained ventricular tachycardia during the hospital stay. Due to high risk for deadly arrhythmias, the patient underwent single-chamber automatic implantable cardioverterdefibrillator (AICD) placement. He was started on warfarin for long-term anticoagulation due to nearly immobile atriums and ventricles. He was evaluated for heart and liver transplants. The liver transplant was postponed due to his heart condition, and ideally, should be pursued after the heart transplant in order to preserve the transplant heart from amyloid deposition. Due to severe hypertrophy with significantly decreased chamber size, left ventricular assisted device was not feasible. Eventually, he was deemed not to be a candidate for the heart transplant due to increased pulmonary vascular resistance. He is currently on palliative care.

\section{Discussion}

In patients without hypertension or valvular disease, biventricular hypertrophy on TTE should raise suspicion for infiltrative cardiomyopathy particularly when associated with low voltage on EKG, as hypertensive left ventricular hypertrophy or hypertrophic cardiomyopathy will usually have high/normal QRS voltage. The cardiac MRI with late gadolinium enhancement is very helpful in supporting the diagnosis of amyloid cardiomyopathy with a sensitivity of $80 \%$ and specificity of $94 \%$, positive and negative predictive values of $92 \%$ and $85 \%$, respectively [7]. Endomyocardial biopsy is the biopsy of choice for diagnosing ATTR, due to high rates of negative biopsies elsewhere [2]. After histologic confirmation of amyloidosis, if special immunostaining to confirm amyloid subtype is not available, serum and urine immunofixation, immunoglobulin free light chain assay, and bone marrow biopsy are needed to exclude amyloid light-chain (AL) amyloidosis. Further TTR mutation sequencing analysis will differentiate familial from senile type ATTR. In terms of treatment, diuretics use is complicated in ATTR cardiomyopathy as stiff heart is often preload dependent. Beta blockers, angiotensinconverting-enzyme (ACE) inhibitors/ angiotensin receptor blockers (ARBs) should be used with caution because they are often poorly tolerated due to hypotension [2]. Early prophylactic AICD placement is rational, given the high incidence of sudden death from arrhythmia and electrical mechanical dissociation [8-9]. Heart and/or liver transplantation are the most important therapeutic interventions. Liver transplantation can be curative in selective patients as it removes the nidus of mutant TTR and prevents recurrent amyloid deposition. More research is needed to evaluate potential pharmacological agents on stabilizing amyloidosis [2].

\section{Conclusions}

We presented a rare case of familial ATTR cardiomyopathy. Unfortunately, by the time of definitive diagnosis, the patient was no longer a candidate for heart or liver transplant, which are potentially curative interventions. Assessment of African American or Afro-Caribbean patients with unexplained heart failure should include a differential diagnosis of transthyretin amyloidosis, as early diagnosis is important to initiate appropriate therapeutic interventions.

\section{Additional Information \\ Disclosures}

Human subjects: Consent was obtained by all participants in this study. Conflicts of interest: In compliance with the ICMJE uniform disclosure form, all authors declare the following:

Payment/services info: All authors have declared that no financial support was received from any organization for the submitted work. Financial relationships: All authors have declared that they have no financial relationships at present or within the previous three years with any 
organizations that might have an interest in the submitted work. Other relationships: All authors have declared that there are no other relationships or activities that could appear to have influenced the submitted work.

\section{References}

1. Benson MD, Dasgupta NR: Amyloid cardiomyopathy. J Am Coll Cardiol. 2016, 68:25-28. 10.1016/j.jacc.2016.05.009

2. Gertz MA, Benson MD, Dyck PJ, et al.: Diagnosis, prognosis and therapy of transthyretin amyloidosis. J Am Coll Cardiol. 2015, 66:2451-2466. 10.1016/j.jacc.2015.09.075

3. Merlini G, Bellotti V: Molecular mechanisms of amyloidosis. N Engl J Med. 2003, 349:583596. 10.1056/NEJMra023144

4. Falk RH: The neglected entity of familial cardiac amyloidosis in African Americans . Ethn Dis. 2002, 12:141-143.

5. Connors LH, Doros G, Sam F, et al.: Clinical features and survival in senile systemic amyloidosis: comparison to familial transthyretin cardiomyopathy. Amyloid. 2011, 18:157159. 10.3109/13506129.2011.574354059

6. Connors LH, Prokaeva T, Lim A, et al.: Cardiac amyloidosis in African Americans: comparison of clinical and laboratory features of transthyretin V122I amyloidosis and immunoglobulin light chain amyloidosis. Am Heart J. 2009, 158:607-614. 10.1016/j.ahj.2009.08.006

7. Vogelsberg H, Mahrholdt H, Deluigi CC, et al.: Cardiovascular magnetic resonance in clinically suspected cardiac amyloidosis: noninvasive imaging compared to endomyocardial biopsy. J Am Coll Cardiol. 2008, 51:1022-1030. 10.1016/j.jacc.2007.10.049

8. Lin G, Dispenzieri A, Kyle R, et al.: Implantable cardioverter defibrillators in patients with cardiac amyloidosis. J Cardiovasc Electrophysiol. 2013, 24:793-798. 10.1111/jce.12123

9. Gregoratos G, Abrams J, Epstein AE, et al.: 2002 guideline update for implantation of cardiac pacemakers and anti arrhythmia devices: summary article: a report of the American College of Cardiology/American Heart Association Task Force on Practice Guidelines (ACC/AHA/NASPE committee to update the 1998 pacemaker guidelines). Circulation. 2002, 106:2145-2161. 